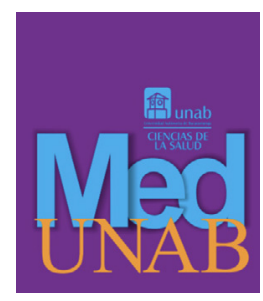

REVISTA DE LA FACULTAD

DE CIENCIAS DE LA SALUD

\title{
Hemicolectomía radical laparoscópica: opción de mínima invasión para el cáncer de colon
}

Laparoscopic radical hemicolectomy: minimally invasive option for colon cancer

Hemicolectomia radical laparoscópica: opcão minimamente invasiva para cãncer de cólon

Luis Ernesto López-Gómez, MD, Esp. ${ }^{1}$ (D), Gonzalo Andrés Dominguez-Alvarado $\mathrm{Md}^{2}$ (D), Daniela D'vera Camargo, Est. ${ }^{3}$ (D), Luis Alejandro Lozano-Eslava, $\mathrm{Md}^{4}{ }^{4}$ (D), Paula Andrea Martínez-Rojas, Est. ${ }^{3}$ (D)

1. Médico, Especialista en Cirugía General y Oncológica. Universidad Autónoma de Bucaramanga, Fundación Oftalmológica de Santander - Clínica Ardila Lülle FOSCAL, Centro de Cáncer, Centro Élite, Unidad especializada en Sobrepeso y Obesidad. Bucaramanga, Santander, Colombia.

2. Médico Servicio Social Obligatorio, Clínica Foscal Internacional, Coordinador Semillero de Innovación e Investigación en Cirugía (SIIC), Floridablanca, Santander, Colombia.

3. Estudiante Medicina, Universidad Autónoma de Bucaramanga, Bucaramanga, Santander, Colombia.

4. Médico General, Universidad Autónoma de Bucaramanga, Bucaramanga, Santander, Colombia.

Correspondencia. Gonzalo Andrés Domínguez Alvarado. Foscal Internacional, Torre C, nivel 4 consultorio 403, Floridablanca, Santander, Colombia. Email. cirugía.semillero@gmail.com

\section{INFORMACIÓN DEL ARTÍCULO:}

Artículo recibido: 20 de noviembre 2019

Artículo aceptado: 10 de mayo 2020

DOI: https://doi.org/10.29375/01237047.3829

Cómo citar: López-Gómez LE, Dominguez - Alvarado GA, D’vera Camargo D, Lozano-Eslava LA, MartínezRojas PA. Hemicolectomía radical laparoscópica: opción de mínima invasión para el cáncer de colon. MedUNAB. 2020;23(2): 281-287. doi: 10.29375/01237047.3829

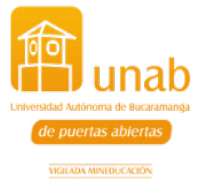

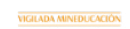

\section{RESUMEN}

Introducción. El cáncer de colon es una de las principales causas de morbimortalidad a nivel mundial. En Colombia se presentan cerca de 145,600 casos nuevos al año. Cada vez son más los reportes de este tipo de patologías intervenidas por vía laparoscópica, aunque son pocos en Colombia. El objetivo de este artículo es mostrar el abordaje de esta patología mediante un procedimiento mínimamente 
invasivo: la colectomía derecha por vía laparoscópica, el cual es poco utilizado en el país. Caso clínico. Paciente de 86 años que consulta por pérdida de peso, adinamia y dolor abdominal en flanco derecho. Presenta además anemia ferropénica y sangre oculta en materia fecal. Las endoscopias digestivas muestran lesión ulcerada vegetante de aspecto neoplásico a nivel del ciego. La biopsia revela adenocarcinoma infiltrante moderadamente diferenciado con áreas de necrosis. Se realiza una colectomía derecha por técnica laparoscópica. Se hace seguimiento con neoadyuvancia por parte de Oncología Clínica. Discusión. El adenocarcinoma es uno de los cánceres primarios más comunes en el colon. La resección quirúrgica es una excelente alternativa para el manejo y tratamiento de estos tumores. En Colombia, la mayoría de estas resecciones se realizan por vía abierta. La resección por vía laparoscópica es un método que demuestra similar eficacia, ofreciendo una disminución en la respuesta inflamatoria, con mejor control del dolor y limitaciones funcionales. Conclusiones. La colectomía por vía laparoscópica para resección de tumores de colon es un método seguro, eficaz y reproducible. Sin embargo, exige tecnología de alto costo y debe ser realizada por un grupo quirúrgico con habilidad y experiencia en sutura manual laparoscópica.

Palabras claves:

Colectomía; Laparoscopía; Enfermedades del Colon; Procedimientos Quirúrgicos Mínimamente Invasivos; Neoplasias del Colon; Neoplasias del Recto.

\section{ABSTRACT}

Introduction. Colon cancer is one of the main causes of morbimortality in the world. Colombia counts near 145,600 new cases per year. The reports of laparoscopic intervention in this pathology are growing, but in Colombia there is little recent work on the matter. The aim of this article is to show how this pathology can be addressed through a minimally invasive procedure, laparoscopic right colectomy, which is not frequently used in the country. Clinical case. An 86-year-old patient consults due to weight loss, adynamia and abdominal pain on right side. Iron-deficiency anemia and hidden blood in feces was reported. The digestive endoscopies show the presence of a vegetant and ulcerated lesion of neoplastic appearance in the cecum. A biopsy revealed a moderately differentiated, invasive adenocarcinoma with areas of necrosis. A laparoscopic right colectomy is conducted. Definitive report of moderately differentiated and ulcerated, invasive, mucinous adenocarcinoma, with lesion up to the serous membrane. Follow up is conducted with neoadjuvant therapy by the Clinical Oncology service. Discussion. Adenocarcinoma is one of the most common primary cancers in the colon. Surgical still an excellent alternative for the management of this kind of tumor. In Colombia, the majority of these resections are carried out by open surgery. However, laparoscopic resection is a method that offers similar outcomes to the traditional procedure, while providing a reduction in the inflammatory response, with better pain control and control of functional limitations. Conclusions. Laparoscopic colectomy for the resection of colonic tumors is a safe, effective and reproducible method. However, it requires high-cost technology and it must be conducted by a surgical team skilled and experienced in manual laparoscopic suturing.

Keywords:

Colectomy; Laparoscopy; Colonic Diseases; Minimally Invasive Surgical Procedures; Colonic Neoplasms; Rectal Neoplasms.

\section{RESUMO}

Introdução. $\mathrm{O}$ câncer de cólon é uma das principais causas de morbimortalidade em todo o mundo. Na Colômbia, há uma incidência aproximada de 145,600 casos novos por ano. Na Colômbia existam poucos trabalhos recentes sobre este tipo de procedimentos. O objetivo deste artigo é mostrar a abordagem de o adenocarcinoma de cólon, através de um procedimento minimamente invasivo: a colectomia direita pela via laparoscópica, pouco utilizada no país. Caso clínico. Paciente de 86 anos que apresenta perda de peso, adinamia e dor abdominal no flanco direito, anemia ferropriva e sangue oculto nas fezes. As endoscopias digestivas evidenciam a presença de uma lesão ulcerada vegetante com aparência neoplásica no nível do ceco. A biópsia revelou adenocarcinoma infiltrante moderadamente diferenciado com áreas de necrose. As imagens de extensão mostram um efeito de massa intraluminal 
ao nível do ceco com adenomegalia pericecal. Realizou-se uma colectomia direita laparoscópica. Relato definitivo de adenocarcinoma mucinoso infiltrante moderadamente diferenciado e ulcerado, com comprometimento da serosa. O serviço de Oncologia Clínica fez o acompanhamento neoadjuvante. Discussão. $\mathrm{O}$ adenocarcinoma é um dos cânceres primários mais comuns no cólon. A ressecção cirúrgica continua sendo uma excelente alternativa para a gestão e tratamento deste tipo de tumores. Na Colômbia, a maioria dessas ressecções é realizada pela via aberta. A ressecção pela via laparoscópica é um método que oferece ressecções semelhantes às do procedimento tradicional, apresentando diminuição da resposta inflamatória, com melhor controle da dor e das limitações funcionais. Conclusão. A colectomia pela via laparoscópica para ressecção de tumores de cólon é um método seguro, eficaz e reprodutível. No entanto, requer tecnologia de alto custo e deve ser realizada por um grupo cirúrgico com habilidade e experiência em sutura manual laparoscópica.

Palavras-chave:

Colectomia; Laparoscopia; Doenças do Cólon; Procedimentos Cirúrgicos Minimamente Invasivos; Neoplasias do Cólon; Neoplasias Retais.

\section{Introducción}

El cáncer es la segunda causa de muerte en todo el mundo. En el 2015 se atribuyeron 8,8 millones de defunciones a esta enfermedad, siendo el cáncer colorrectal (CCR) el cuarto dentro de este grupo con 788,000 defunciones (1). En Estados Unidos de América el cáncer de intestino grueso tiene una incidencia de 145,600 casos nuevos por año y es la tercera causa de muerte. Para el 2012, según Globocan (2), el CCR ocupó en Colombia el quinto lugar en los tumores malignos más frecuentes para ambos sexos con una tasa de incidencia de 12.9 casos por cada 100,000 habitantes y tuvo el mismo lugar de frecuencia en mortalidad con tasas hasta de 7.2 casos por cada 100,000 habitantes. En Bucaramanga y su área metropolitana el CCR tiene una incidencia de 14.3 casos en hombres y 13.5 casos en mujeres por 100,000 habitantes, y la edad promedio de diagnóstico está alrededor de los 64 años para ambos sexos (3-5).

Sus síntomas se asocian principalmente con la ubicación tumoral y dependen del grado de compromiso de este. También pueden ser asintomáticos y se descubren mediante estudios de rutina, con síntomas menores confusos. Algunas veces, los pacientes ingresan al servicio de urgencias con signos de obstrucción intestinal, peritonitis $y$, en raras ocasiones, con sangrado gastrointestinal agudo. Sin embargo, los síntomas y signos más típicos asociados a este cáncer incluyen: hematoquecia o melenas, dolor abdominal, anemia crónica por deficiencia de hierro inexplicable o un cambio en los hábitos intestinales $(6,7)$.

En cuanto al diagnóstico del cáncer de colon la US Preventive Services Task Force recomienda iniciar la detección temprana del mismo en pacientes de 50 años o más que tengan riesgo medio de padecer CCR o que presenten síntomas sugestivos. Con este fin se debe iniciar un diagnóstico por medio de una sospecha clínica de uno o más de los signos y síntomas anteriormente descritos. Si existe sospecha, se debe realizar una colonoscopia o una Tomografía Computarizada (TC) para confirmar el diagnóstico. La colonoscopia actualmente sigue siendo el gold standard para el diagnóstico de cáncer colorrectal $(8,9)$.

Debido a la tendencia ascendente en la incidencia de adenocarcinoma de colon en el mundo (y en Colombia), así como la disponibilidad de nuevas técnicas para su resección, se considera necesario exponer un caso exitoso en el manejo de esta patología, realizando la resección tumoral por colectomía derecha vía laparoscópica, cuyo éxito promueva la instauración de abordajes menos invasivos y con menor tasa de comorbilidades en los pacientes.

\section{Caso clínico}

Paciente femenina de 86 años con antecedentes de hipertensión arterial y gastritis crónica atrófica, con cuadro de seis meses de evolución de dolor abdominal en flanco derecho, adinamia y pérdida de peso no cuantificada, motivo por el cual consulta a medicina general, desde donde se remite una colonoscopia que evidencia lesión proliferativa infiltrante amplia a nivel de tercio proximal colon ascendente (Figura 1). Resultado histopatológico de adenocarcinoma de patrón clásico moderadamente diferenciado infiltrante. Estudio complementario con TC abdominal que demuestra engrosamiento infiltrativo de las paredes del ciego, de probable naturaleza neoplásica con compromiso de la unión ileocecal y de ganglios pericecales (Figura 2). Los paraclínicos prequirúrgicos (Tabla 1) informaron anemia moderada. Valorada por cirugía general en donde se decide realizar tratamiento quirúrgico Hemicolectomía derecha radical. 
Figura 1. Colonoscopia, a nivel del colon ascendente tercio proximal lesión proliferativa infiltrante, amplia, en media luna de consistencia dura y con resultado histopatológico de adenocarcinoma de patrón clásico moderadamente diferenciado infiltrante
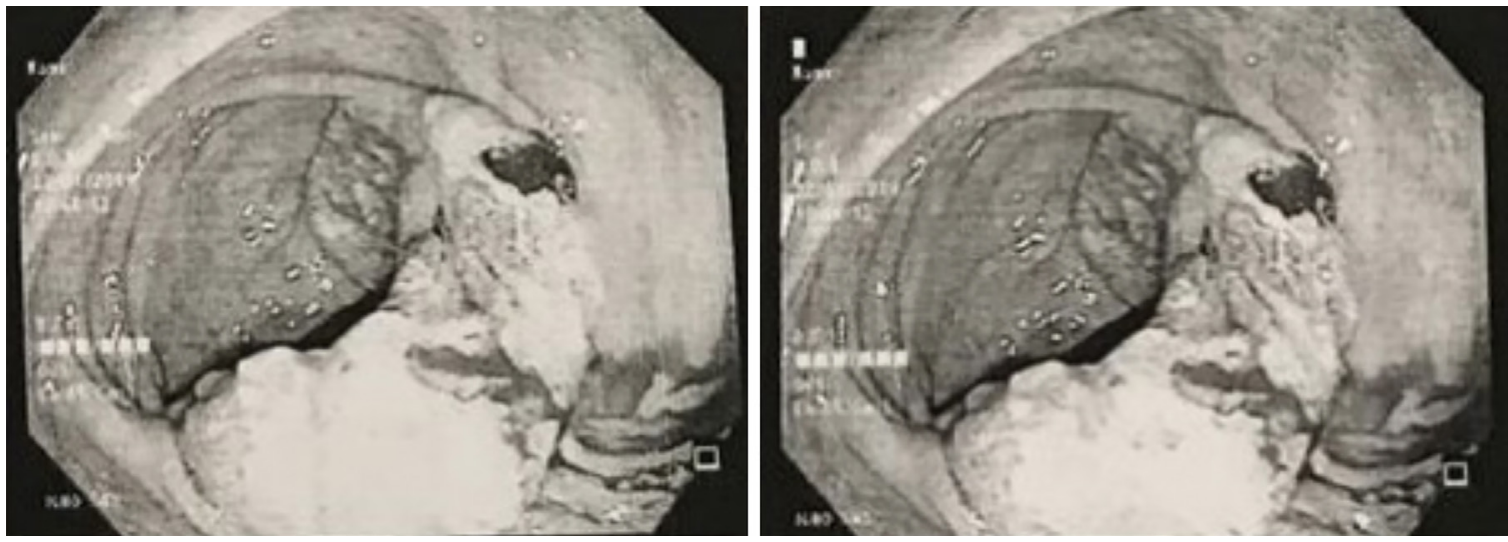

Fuente: Historia clínica del paciente

Figura 2. TAC con engrosamiento infiltrativo de las paredes del ciego de probable naturaleza neoplásica, que compromete la unión ileocecal y afecta ganglios pericecales
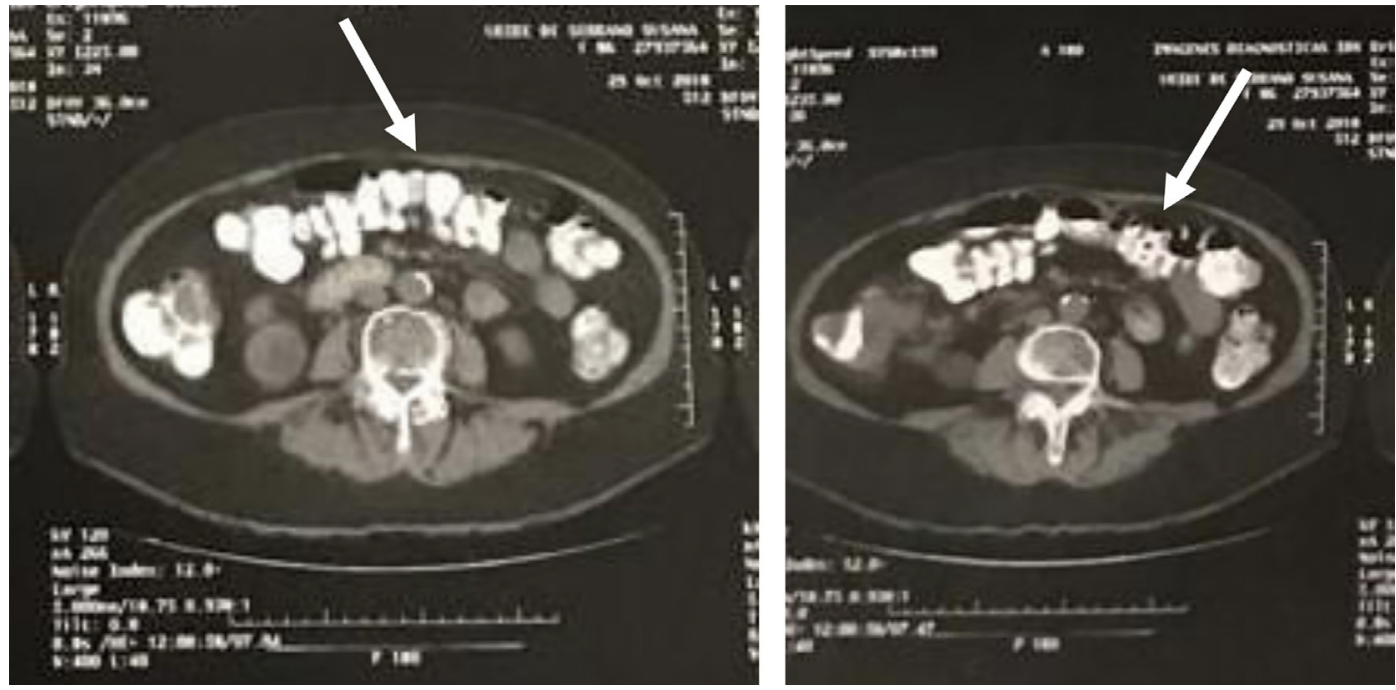

Fuente: Historia clínica del paciente

Tabla 1. paraclínicos prequirúrgicos.

\begin{tabular}{c} 
Laboratorio \\
\hline Glóbulos rojos \\
\hline Hemoglobina \\
\hline Hematocrito \\
Serie leucocitaria \\
Serie plaquetaria \\
\hline Tiempo de Protrombina (PT) \\
\hline Tiempo parcial de tromboplastina (PTT) \\
\hline Nitrógeno ureico \\
Creatinina
\end{tabular}

Fuente: Elaborada por los autores

$\begin{array}{cc}\text { Valor paciente } & \text { Valores normales } \\ 3,590.000 / \mathrm{mm} 3 & 4,500.000-5,900.000 / \mathrm{mm} 3 \\ 9.5 \mathrm{gr} / \mathrm{dl} & 14.0-17.5 \mathrm{gr} / \mathrm{dl} \\ 29.6 \% & 40-52 \% \\ 9,210 / \mathrm{mm} 3 & 4,400-11,300 / \mathrm{mm} 3 \\ 371,000 / \mathrm{mm} 3 & 150,000-440,000 / \mathrm{mm} 3 \\ 10.3 \mathrm{segundos} & 9.7-13.5 \mathrm{seg} \\ 24.5 \mathrm{segundos} & 22.5-30.5 \mathrm{seg} \\ 14.3 \mathrm{mg} / \mathrm{dl} & 8.0-23.0 \mathrm{mg} / \mathrm{dl} \\ 0.79 \mathrm{mg} / \mathrm{dl} & 0.67-1.17 \mathrm{mg} / \mathrm{dl}\end{array}$


Con respecto a la técnica quirúrgica, se practica una exploración laparoscópica por puerto de visión ubicado a nivel umbilical. Se identifica una masa tumoral maligna a nivel de colon ascendente (Figura 3). Macroscópicamente no se observa tumor en la serosa. Al comprobar que no existen contraindicaciones para continuar con esta técnica y que la lesión es resecable, se procede a colocar otros dos puertos, todos en línea media. El autor utiliza la línea media como medida para evitar pasar por los músculos de la pared abdominal y así evitar el dolor y sangrado postoperatorio. El equipo quirúrgico está ubicado al lado izquierdo del paciente y la torre de laparoscopia al lado derecho. Se practica una incisión en fascia de Toldt con la disección clásica de maniobra de Catell y con movilización medial del colon derecho hasta visualizar el riñón derecho, el duodeno, el uréter derecho y la vena cava inferior. De esta manera se garantiza la radicalidad del procedimiento. Esta maniobra levanta el mesocolon derecho hasta la raíz del mesenterio, dando facilidad a la identificación de la arteria cólica derecha. Esta, junto con su vena, se secciona entre clips metálicos. Después de la liberación del ángulo hepático del colon, la disección se lleva hasta el colon transverso a nivel de la cólica media, la cual se preserva. Se disecan los límites de resección dados por el íleon distal y el colon transverso. Se efectúa resección con endograpadora lineal de $45 \mathrm{~mm}$, recarga de $2.5 \mathrm{~mm}$ para tejido normal. Un corte en el íleon y otro a nivel del colon transverso. La pieza quirúrgica se introduce en bolsa de manejo de tejidos intracorpóreos (Endo Bag) con lo cual se evita contaminación neoplásica de otros tejidos, especialmente a nivel del puerto de salida.

Figura 3. Masa tumoral maligna a nivel de colon ascendente sobre la flexura del ángulo hepático del colon.

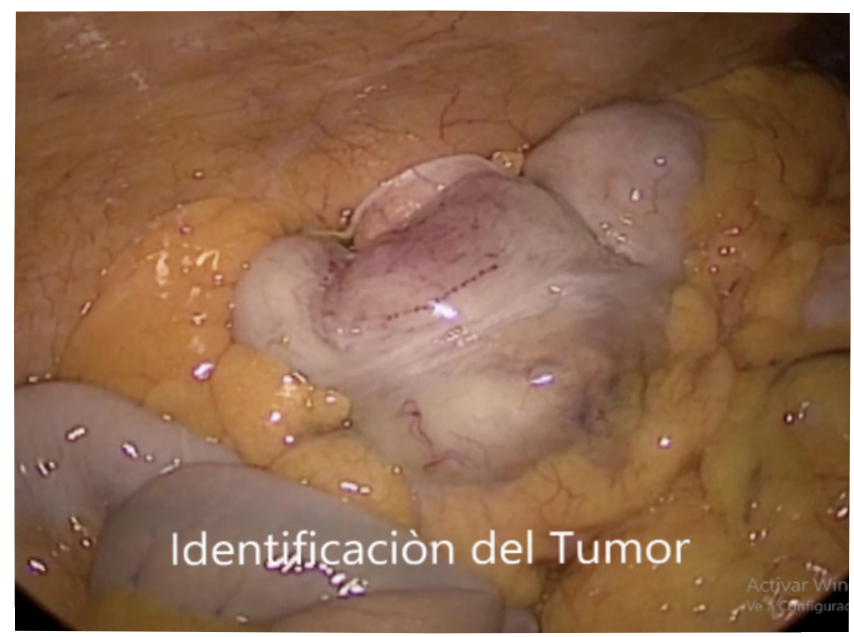

Fuente: Historia clínica del paciente
Se procede a efectuar la anastomosis intracorpórea isoperistáltica con endograpadora lineal de $45 \mathrm{~mm} \mathrm{x}$ 2.5 , una línea de grapado y cierre del orificio con sutura continua en dos planos de polidioxanona de calibre $3 / 0$. Puntos separados de refuerzo en los extremos de la línea de grapa y cierre del defecto de los mesenterios. Finalmente se hace la extracción de la pieza quirúrgica (previamente aislada dentro de la bolsa laparoscópica) por una prolongación de incisión a nivel del puerto umbilical y se cierran los puertos. La duración del procedimiento es de dos horas y media. El sangrado operatorio es considerado mínimo. No se requieren transfusiones. Después de cuatro días de postoperatorio se da de alta hospitalaria a la paciente con adecuada funcionalidad.

\section{Discusión}

Dentro de las neoplasias primarias con origen en el colon, la variedad histopatológica más frecuente es el adenocarcinoma, que tiene una incidencia en hombres de 13.5 y en mujeres 11.9 por cada 100,000 habitantes en Sudamérica. En Colombia, de 12.2 en hombres y 12.3 en mujeres (según el Instituto Nacional de Cancerología-E.S.E del 2011), de los cuales anualmente mueren 1,168 hombres y 2,784 mujeres por esta causa, siendo de gran importancia su estudio (10).

Actualmente los dos métodos más utilizados para el diagnóstico de cáncer colorrectal son la colonoscopia y la TC de colon. Sin embargo, la colonoscopia sigue siendo el gold standard, ya que es la prueba más precisa y versátil además de que permite la toma de muestra para estudio histológico (9).

Una vez establecido el diagnóstico se debe estadificar la lesión tumoral mediante el sistema para estadiaje de tumores: Tumor, Node -nódulo, ganglio- y Metástasis (TNM), con el fin de determinar su extensión y definir un posible manejo quirúrgico. En este caso, la paciente se encontraba en estadio IIIA. Según la American Cancer Society, el manejo indicado en este estadiaje es la resección quirúrgica con quimioterapia adyuvante (11).

En cuanto a la modalidad de manejo primario, la resección quirúrgica ha sido uno de los métodos más eficaces para el tratamiento curativo del cáncer de colon localizado. Su objetivo primordial es la resección completa del tumor y sus áreas de extensión, incluyendo cadenas ganglionares y vías de drenaje venoso, o resección en bloque de estructuras contiguas en caso de infiltración del tumor en un órgano $(10,12)$. Para este fin 
se han descrito diferentes técnicas quirúrgicas, dentro de las cuales se discuten la cirugía convencional abierta versus la colectomía por laparoscopia. El objetivo de ambas es la resección total de la masa tumoral, de sus drenajes vasculares, linfáticos y de los tejidos vecinos, obligando a la reconstrucción funcional del órgano o segmento extirpado. Esta característica es lo que le da el criterio de radicalidad. El abordaje intraabdominal es el mismo para las dos técnicas desde el punto de vista oncológico (márgenes de resección, longitud de la pieza quirúrgica, ligadura alta de los pedículos vasculares, seguridad de la anastomosis y la no manipulación del tumor). Ambas técnicas son actualmente aceptadas y utilizadas ampliamente para manejo de adenocarcinoma de colon (13).

La evidencia actual es suficiente para apoyar y recomendar el uso de la laparoscopia como modalidad quirúrgica la resección de cáncer de colon, ya que ofrece mejores resultados a corto plazo y, al menos, resultados oncológicos a largo plazo comparables con la resección abierta (14). En Colombia, la cirugía convencional abierta para el tratamiento quirúrgico del cáncer colorrectal ha sido la más utilizada. Sin embargo, con el desarrollo tecnológico, la cirugía laparoscópica se ha posicionado cada vez más como el tratamiento de las enfermedades gastrointestinales. En este caso, mostrando beneficios en comparación a la cirugía convencional abierta en pacientes sometidos a hemicolectomía. Por ejemplo, metaanálisis publicados en los últimos años evidencian que el retorno a la dieta fue más rápido $(4.5 \pm 1.2$ días versus $5.4 \pm 1.8$ días, $\mathrm{P}=0.013)$; la estadía hospitalaria postoperatoria fue más corta $(12.1 \pm 4.2$ días versus $15.9 \pm 4.8$ días, $\mathrm{P}=$ 0.000 ); la duración de la incisión, la pérdida de sangre, la movilización temprana después de la cirugía y la tasa de complicaciones generales postoperatorias a los 30 días fueron significativamente más cortas; así como un tiempo más corto hasta el primer flato y mejores puntuaciones de calidad de vida en apetito, insomnio, dolor, fatiga, vida diaria y salud, en comparación con los pacientes que se sometieron a cirugía abierta a los dos días, dos semanas y dos meses después de la operación (15-17). Lo anterior, se relaciona con la paciente acá referida, ya que retornó y realizó movilización al segundo día, flatos al tercer día y egreso hospitalario al cuarto día, sin complicaciones postquirúrgicas.

La cirugía laparoscópica tiene una tasa de complicaciones graves muy baja, dentro de las cuales se encuentran complicaciones intraoperatorias tales como punción accidental durante el acceso abdominal para la colocación de la cámara o el puerto, representando menos del $1 \%$. Otras complicaciones que pueden surgir son la insuflación abdominal, disección de tejidos, hemostasia o la necesidad de conversión a un procedimiento abierto. Las complicaciones graves, como la lesión vascular y la perforación intestinal, que inciden desfavorablemente en el pronóstico del paciente son la principal causa de morbimortalidad específicas del procedimiento relacionadas con esta técnica $(18,19)$. A pesar de lo anterior, la cirugía laparoscópica muestra resultados superiores a la cirugía abierta tanto en estancia hospitalaria, infección de herida y calidad de vida en el primer mes postoperatorio (20).

La cirugía colorrectal laparoscópica es técnicamente desafiante y la evidencia sugiere que existe una curva de aprendizaje pronunciada. Algunos autores como Schlachta y colaboradores mostraron que sólo después de la realización de 30 colectomías laparoscópicas, el tiempo operatorio, las tasas de complicaciones intraoperatorias y la necesidad de conversión a cirugía abierta disminuían (21). Por esto es importante contar con un equipo experimentado que se traduzca en una mayor tasa de éxito, como sucedió en este caso, ya que el autor cuenta con experiencia de aproximadamente 20 años y más de 200 casos de realización de procedimientos quirúrgicos oncológicos en colón por técnica abierta. Desde el año 2005 a la fecha ha realizado más de 70 procedimientos por técnica laparoscópica a nivel de colon y recto, con una frecuencia aproximada de 6 a 8 procedimientos por año.

\section{Conclusión}

A pesar de que actualmente en Colombia la gran mayoría de colectomías se siguen realizando por vía abierta, se considera, según lo reportado en este caso, que la técnica por vía laparoscópica de colectomía derecha para resección del adenocarcinoma de colon es un método que supera al procedimiento tradicional, ya que, de acuerdo con la literatura, es un método seguro, eficaz y reproducible. Sin embargo, el procedimiento debe ser realizado por un grupo quirúrgico con habilidad y experiencia en sutura manual laparoscópica avanzada para evitar posibles complicaciones intra y postoperatorias, y así lograr resultados óptimos.

\section{Referencias}

1. Plummer M, de Martel C, Vignat J, Ferlay J, Bray F, Franceschi S. Global burden of cancers attributable to infections in 2012: a synthetic analysis. Lancet Glob Heal. 2016; 4(9):609-16.

2. Bray F, Ren JS, Masuyer E, Ferlay J. Global 
estimates of cancer prevalence for 27 sites in the adult population in 2008. Int J Cancer. 2013 [cited 2019 Sep 20];132(5):1133-45.

3. Siegel R. L, Miller K.D, Jemal A. Cancer statistics, 2019. CA Cancer J Clin [Internet]. 2019;69(1):734. Retrieved from: http://doi.wiley.com/10.3322/ caac. 21551

4. Antoni S, Soerjomataram I, Møller B, Bray F, Ferlay J. An assessment of GLOBOCAN methods for deriving national estimates of cancer incidence. Bull World Health Organ [Internet]. 2016 [cited 2019 Sep 20];94(3):174-84. Retrieved from: http://www.who. int/entity/bulletin/volumes/94/3/15-164384.pdf

5. Uribe-Pérez CJ, Blanco-Quintero JJ, Bello-Zapata LM. Incidencia de cáncer de colon y recto en Bucaramanga, Colombia 2008 - 2012. MedUNAB. 2019;22(1):16-23.

6. Saidi HS, Karuri D, Nyaim EO. Correlation of clinical data, anatomical site and disease stage in colorectal cancer. East Afr Med J [Internet]. 2008 [cited 2019 Sep 20];85(6):259-62. Retrieved from: http://www. ncbi.nlm.nih.gov/pubmed/18817021

7. Macrae FA, St John DJ. Relationship between patterns of bleeding and Hemoccult sensitivity in patients with colorectal cancers or adenomas. Gastroenterology [Internet]. 1982[cited 2019 Sep 20];82(5 Pt 1):8918. Retrieved from: http://www.ncbi.nlm.nih.gov/ pubmed/7060910

8. Liu GW. CQ-C. Screening for colorectal. World Chinese J Dig [Internet]. 2016; Retrieved from: http:// jamanetwork.com/journals/jama/fullarticle/2529492

9. Atkin W, Dadswell E, Wooldrage K, Kralj-Hans I, Von Wagner C, Edwards R., et al. Computed tomographic colonography versus colonoscopy for investigation of patients with symptoms suggestive of colorectal cancer (SIGGAR): A multicentre randomised trial. Lancet [Internet]. 2013;381(9873):1194-202. Retrieved from: http://dx.doi.org/10.1016/S01406736(12)62186-2

10. Instituto Nacional de Cancerología. Proyecto Guías de Atención Integral (GAI) de cáncer en adultos. [Internet] 2011;4-7. Retrieved from: https://www.minsalud.gov.co/salud/Documents/ PRESENTACIONGUIASCANCEROLOGICO.pdf

11. American Cancer Society. Tratamiento del cáncer de colon según la etapa [Internet]. [cited 2020 Jan 20]. Retrieved from: https://www.cancer.org/es/cancer/ cancer-de-colon-o-recto/tratamiento/por-etapascolon.html

12. Weiser MR, Gon̈en M, Chou JF, Kattan MW, Schrag D. Predicting survival after curative colectomy for cancer: Individualizing colon cancer staging. J Clin Oncol. 2011;29(36):4796-802.

13. Dong B, Luo Z, Lu J, Yang Y, Song Y, Cao J, et al.
Single-incision laparoscopic versus conventional laparoscopic right colectomy: A systematic review and meta-analysis. Int J Surg [Internet]. 2018;55:318. Retrieved from: https://doi.org/10.1016/j. ijsu.2018.05.013

14. Mathis KL, Nelson H. Controversies in laparoscopy for colon and rectal cancer. Surg Oncol Clin N Am. 2014;23(1):35-47.

15. Cirocchi R, Campanile F, Saverio S, Popivanov G, Carlini L, Pironi D, et al. Laparoscopic versus open colectomy for obstructing right colon cancer: A systematic review and meta-analysis. J Visc Surg [Internet]. 2017;154(6):387-99. Retrieved from: http://dx.doi.org/10.1016/j.jviscsurg.2017.09.002

16. McCombie AM, Frizelle F, Bagshaw PF, Frampton CM, Hewett PJ, McMurrick PJ, et al. The ALCCaS Trial: A randomized controlled trial comparing quality of life following laparoscopic versus open colectomy for colon cancer. Dis Colon Rectum. 2018;61(10):1156-62.

17. Kim MK, Won DY, Lee JK, Kang WK, Kye BH, Cho HM., et al. Laparoscopic Surgery for Transverse Colon Cancer: Short- and Long-Term Outcomes in Comparison with Conventional Open Surgery. J Laparoendosc Adv Surg Tech. 2015;25(12):982-9.

18. Vogel JD, Eskicioglu C, Weiser MR, Feingold DL, Steele SR. The American society of colon and rectal surgeons clinical practice guidelines for the treatment of colon cancer. Dis Colon Rectum. 2017;60(10):9991017.

19. Zheng Z, Jemal A, Lin CC, Hu CY, Chang GJ. Comparative effectiveness of laparoscopy vs open colectomy among nonmetastatic colon cancer patients: An analysis using the national cancer data base. J Natl Cancer Inst. 2015;107(3):13-5.

20. Balén E, Suárez J, Ariceta I, Oronoz B, Herrera J, Lera JM. Cirugía laparoscópica en las enfermedades colorrectales. An Sist Sanit Navar. 2005;28.

21. Schlachta CM, Mamazza J, Seshadri PA, Cadeddu M, Gregoire R, Poulin EC. Defining a learning curve for laparoscopic colorectal resections. Dis Colon Rectum. 2001;44(2):217-22. 